\title{
ON STEADY TWO-DIMENSIONAL FREE-SURFACE FLOWS WITH SPATIALLY-VARIED DISCHARGES
}

Yebegaeshet T. ZERIHUN ${ }^{1 *}$

\section{Abstract}

In an open-channel, the transition of a flow from a subcritical to a supercritical state may occur as a result of a lateral inflow or outflow that produces a streamwise discharge variation. Apparently, such a transition cannot be modeled accurately by a conventional hydrostatic pressure approach. In this study, a depth-averaged model that accounts for the effects of a spatially-varied discharge and a non-hydrostatic pressure distribution was developed and applied to simulate the transcritical flow in a lateral-spillway channel and the subcritical flow in a main channel fitted with side weirs. The model results for the axial free-surface profile and variation of discharge in the main channel were compared with the results of a shallow-flow model and experimental data, thereby resulting in a closer match to the measurements than the shallow-flow model. Overall, the investigation results confirmed the efficiency and validity of the non-hydrostatic depth-averaged model in simulating the mean flow characteristics of the subcritical and transcritical free-surface flows with spatially increasing or decreasing discharges, thus demonstrating its potential to be used as a numerical tool in engineering practice.

\section{Address}

1 David \& James - Engineering and Environmental Consultancy, Victoria, Australia

* Corresponding author: zyebegaeshet@gmail.com

\section{Key words}

- Hydraulic structures,

- Side-weir overflow,

- Non-hydrostatic flow,

- Spatially-varied flow,

- Numerical model,

- Side-channel spillway.

\section{INTRODUCTION}

In essence, a steady, spatially-varied flow is a non-uniform flow in which the depth of flow, velocity and discharge vary with respect to space, but are constant with respect to time. The nature of the free-surface profile of the flow depends largely on whether the streamwise discharge is increasing or decreasing. Examples of such a type of flow with a spatially-increasing discharge include a lateral-spillway channel, highways and urban stormwater drainage channels, and effluent channels around sewage treatment tanks. A main channel with side weirs is the most common example of an open-channel flow with a spatially-decreasing discharge. Experimental investigations and theoretical analyses of spatially-varied flows with increasing or decreasing discharges have been of interest to many investigators in the field of open-channel hydromechanics. Experimental studies have been car- ried out to obtain an understanding of the salient features of an axial channel flow as well as a determination of the discharge characteristics of overflow structures (see, e.g., Hinds, 1926; Farney and Markus, 1962; Gill, 1977; Hager, 1982; Bremen and Hager, 1989; Maranzoni et al., 2017). The conventional hydraulic approach, which forms the basis of the theoretical analysis of a spatially-varied flow, assumes that the effects of a vertical acceleration are negligible, so that the flow problem can be treated as a one-dimensional free-surface flow with a spatially-varied discharge. Nonetheless, the numerical results of this modeling approach have often been compared with experimental data in which such an assumption does not strictly remain valid. Any efforts in developing dynamic equations must therefore be based on including more vertical details in the form of the velocity and non-hydrostatic pressure distributions as well as the effects of the channel bed topography. From a practical viewpoint, an accurate open-channel flow model 
can provide useful information for developing design guidelines and/ or assessing the hydraulic performance of side channels and overflow structures as well as the discharge capacity of roof gutters.

Hinds (1926) was probably the first to develop a dynamic equation of a spatially-varied flow with an increasing discharge using the momentum principle along with an approximation of the hydrostatic pressure. In this method, the effects of channel friction and the contribution of the lateral inflow in the streamwise momentum flux were ignored. Later, Favre (1933) applied both the energy and momentum principles, along with assumptions of a uniform velocity and hydrostatic pressure distributions, to develop dynamic equations for cases of a spatially-varied flow with increasing and decreasing discharges. Compared to the Hinds method, Favre's approach led to the inclusion of a friction term. In addition, the contributions of other investigators working in this area, notably De Marchi (1934), Camp (1940), Li (1955), and Liggett (1959), led to improvements in Hinds' original approach. In practice, however, a spatially-varied flow field is characterized by substantial vertical curvatures of the streamline and, hence, by a departure from the distribution of the hydrostatic pressure. As noted by Montes (1998), the effects of such a streamline geometry on flow patterns must be considered for accurately treating the problem of a spatially-varied flow with curved streamlines.

In the past, higher-order approaches that could overcome the inherent limitations of approximating hydrostatic pressure have been proposed. By applying the concepts of the conservation of energy and momentum, Nakagawa (1969) systematically extended the gradually-varied flow equations of non-uniform discharges for a curved free-surface flow with a spatially-decreasing discharge due to a bottom outlet flow. His method allowed for variations of velocity and non-hydrostatic pressure across the flow depth, thereby leading to a higher-order approximation for treating the flow problem. Yen and Wenzel (1970) also developed the energy and momentum forms of spatially-varied flow equations for the case of a steady flow in an open channel with a lateral inflow or outflow. Their equations included unspecified velocity and pressure correction coefficients that made the equations not readily applicable unless the distributions of these correction coefficients were known in advance (Yen, 1971; Yen, 1973; Hager and Volkart, 1986). Balmforth and Sarginson (1983) employed a Boussinesq approximation to analyze free-surface flows over side weirs with supercritical inflow conditions. Hager et al. (1988) applied a Boussinesq-type equation with a spatially-constant discharge to analyze free-surface flow in a lateral-spillway channel. As the method of the derivation ignored the lateral inflow, the resulting equation did not incorporate terms that accounted for the effects of a spatially-varied discharge. Similar to the Balmforth and Sarginson (1983) approach, this method is not rigorous for a spatially-varied flow situation because it contravenes the kinematic boundary condition at the free surface. Following the approach of Nakagawa (1969), Castro-Orgaz and Hager (2011) recently presented dynamic equations for flows with spatially increasing or decreasing discharges. The scope of both studies was limited only to an analysis of the spatially-varied flows in a horizontal bed channel. This implies that the equations of Nakagawa (1969) and Castro-Orgaz and Hager (2011) are not suited for analyzing such types of flows in a channel with varying longitudinal bed topography.

Although there has been considerable progress during recent years in the development of numerical models to explore the mean flow characteristics of a spatially-varied open-channel flow, much less effort has been made in proposing a general-purpose non-hydrostatic model for systematically investigating the problems of a free-surface flow with a spatially increasing or decreasing discharge. Additionally, most of the previous numerical simulation studies were conducted using the conventional hydrostatic pressure approach (see, e.g., Gill, 1977; Hager, 1983; Kouchakzadeh et al., 2001). It is well-known that a flow transition from a subcritical to a supercriti- cal state can be caused by a lateral inflow or outflow that produces a streamwise non-uniform discharge. This reveals that this type of flow exhibits key characteristics that resemble a two-dimensional (2D) plane flow, which makes the tools for the hydrostatic pressure approach less useful. The present study primarily aims at developing a spatially-varied flow equation that incorporates a higher-order dynamic pressure correction for the effects of the vertical curvatures of the streamline by applying the momentum principle and Boussinesq's theory. The formulation of this type of governing equation requires a specification of the kinematic boundary condition on a free surface or bed. For an open-channel flow with a spatially-increasing discharge or a lateral outflow due to a side weir, the free surface is not a streamline, but is a surface along which streamlines begin or terminate. This implies that the same governing equation can be applied to analyze flow situations with a spatially increasing or decreasing (as a result of a side-weir flow) discharge. As a part of this study, the proposed depth-averaged equation will be thoroughly examined to simulate the mean flow characteristics of 2D spatially-varied flows. In an analysis of such types of free-surface flows, a feasible approach is to ignore variations of the flow parameters in the transverse direction so that the complex problem can be treated as a $2 \mathrm{D}$ plane flow problem. Accordingly, the axial free-surface profile can be modeled by computing the cross-sectional average depths along the centerline of the channel. Such an approximate modeling approach permits an assessment of the effect of the streamline vertical curvatures on the free-surface profile and main-channel discharge solutions of the equations. It is also aimed at carrying out a numerical experiment so as to examine quantitatively the contributions of the non-hydrostatic terms of the governing equation by comparing its solutions for curvilinear spatially-varied flow problems with the measurements.

The outline of the paper is as follows. In the following sections, the spatially-varied discharge form of the Boussinesq-type equations is presented, and the main features of the extended computational model, namely the spatial discretization of the equations and the solution procedures for the resulting non-linear discretized equations, are outlined. The boundary conditions are also described with reference to the case studies. A brief discussion of the performance of the model for spatially-varied flow problems is presented by comparing the numerical results with the experimental data from the literature. The paper ends with a summary and discussion.

\section{GOVERNING EQUATIONS}

For a rectangular channel, Cartesian coordinates such that $x$ is horizontally along the channel; $y$ is vertically upward; and $z$ is horizontally in the transverse direction, are defined, as illustrated in Fig. 1. The momentum equation for a free-surface flow in a straight channel can be written as (Fenton and Zerihun, 2007):

$$
\frac{\partial Q}{\partial t}+\beta \frac{\partial}{\partial x}\left(\frac{Q^{2}}{A}\right)+\frac{1}{\rho} \int_{A} \frac{\partial p}{\partial x} d A-\beta_{L} q_{L} U_{L}+\frac{f P_{w} Q^{2}}{8 A^{2}}\left(1+Y_{x}^{2}\right)=0
$$

where $f$ denotes the Darcy-Weisbach friction coefficient; $Q$ is the discharge; $A$ is the cross-sectional area of the flow; $\rho$ is the density of the fluid; $p$ is the pressure; $\beta$ refers to the Boussinesq momentum coefficient; $Y_{x}\left(=-S_{0}\right)$ is the bed slope; $P_{w}$ is the wetted perimeter; $\beta_{L} q_{L} U_{L}$ is the contribution of an inflow or outflow of the $q_{L}$ volume rate per unit length with a streamwise velocity component $U_{L}(=V \cos \theta) ; V$ is the local lateral inflow or outflow velocity; $\theta$ is the lateral inflow or outflow angle; and $t$ is the time. It is important to note that $q_{L}$ is positive for a lateral inflow and is negative for a lateral outflow. 


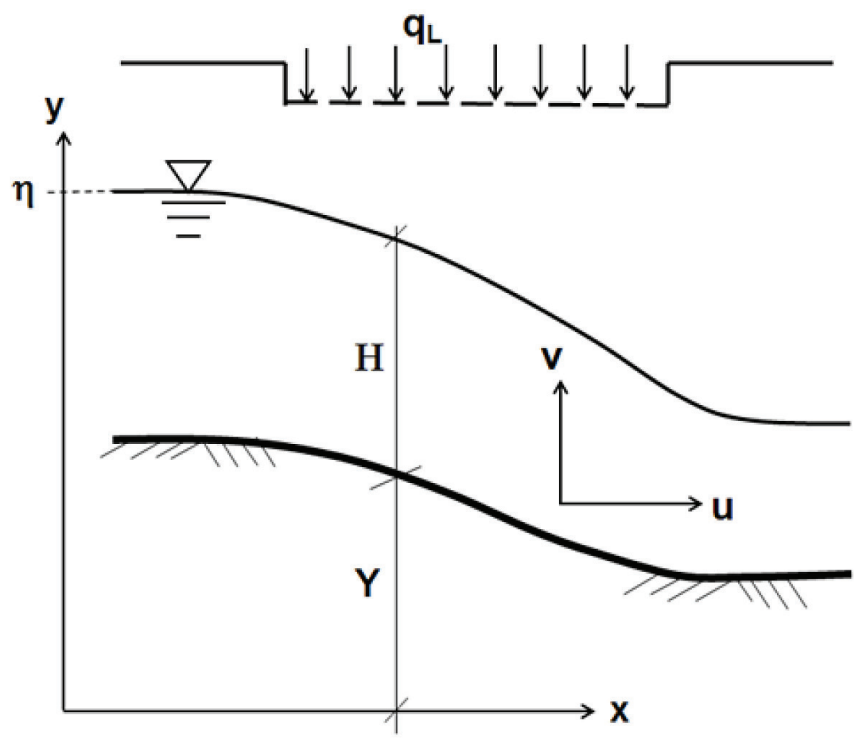

Fig. 1 Definition sketch for a spatially-varied flow with an increasing discharge

The approximate treatment of the effects of the vertical acceleration of the flow requires a pre-defined distribution of centrifugal term across the flow depth. Using different distribution shapes, Zerihun (2004), Zerihun and Fenton (2006), and Fenton and Zerihun (2007) applied this modeling approach to analyze flows with spatially-constant discharges. Herein, the continuity equation, along with a pre-assumed horizontal velocity distribution, is employed to account for the effects of the dynamic pressure and spatially-varied discharges. For a $2 \mathrm{D}$ open-channel flow, the continuity equation reads as

$$
\frac{\partial u}{\partial x}+\frac{\partial v}{\partial y}=0,
$$

where $u$ and $v$ are the velocity components in the horizontal and vertical directions, respectively. Using the lowest-order approximation, the horizontal velocity in a vertical section is given by its depth-averaged value as

$$
u=\frac{q}{H},
$$

where $q$ is the discharge per unit width, and $H$ is the depth of flow. Such an approximation is not uncommon in open-channel flow modeling. As noted by Hager (1982) for side-channel flows, the effect of a horizontal velocity variation in a vertical direction is insignificant compared to its distribution effect in the transverse direction.

Since the inflow or outflow takes place along the free surface at a constant or non-uniform rate, this surface is not a streamline, but is a surface along which the streamlines begin or terminate. Substituting Eq. (3) into Eq. (2) and vertically integrating the resulting expression from the bed to an arbitrary point $y$, the equation for the vertical velocity distribution can be obtained after employing the kinematic boundary condition, $v_{b}=Y_{x} u$, at the bed of the channel, i.e.,

$$
\begin{gathered}
v=-q_{x} \xi+\frac{q}{H} H_{x} \xi+\frac{q}{H} Y_{x}, \\
\xi=\frac{y-Y}{\eta-Y},
\end{gathered}
$$

where $q_{x}$ is the inflow or outflow discharge per unit length per unit width; $v_{b}$ is the vertical velocity at the channel bed; $Y$ is the channel bed elevation; $\eta$ is the mean elevation of the free surface; and $\xi$ is a dimensionless vertical height. Compared to earlier approaches (e.g., Zerihun and Fenton, 2006; Fenton and Zerihun, 2007), Eq. (4) implicitly incorporates the effects of a spatially-varied discharge in the distribution of the vertical velocity.

For a steady, curvilinear free-surface flow $(\partial v / \partial t=0)$, the pressure distribution at a vertical section can be deduced from the integration of Euler's equation as follows (White, 2011):

$$
u \frac{\partial v}{\partial x}+v \frac{\partial v}{\partial y}=G_{y}-\frac{1}{\rho} \frac{\partial p}{\partial y},
$$

where $G_{y}(=-\mathrm{g})$ is the body force per unit mass, and $\partial p / \partial y$ is the pressure gradient in the vertical direction. By multiplying both sides of Eq. (6) by $\partial y$, integrating vertically between an arbitrary point $y$ and the free surface $\eta$, and applying the dynamic boundary condition $(p(\eta)=0)$, one can obtain

$$
\frac{p}{\rho}=-\int_{y}^{\eta} G_{y} d y+\int_{y}^{\eta} u \frac{\partial v}{\partial x} d y+\int_{y}^{\eta} v \frac{\partial v}{\partial y} d y .
$$

Differentiating Eq. (4) with respect to $x$ and then substituting the resulting expression into Eq. (7) results in the pressure distribution equation for flow in a rectangular channel as follows:

$$
\begin{aligned}
& \frac{p}{\rho g}=H(1-\xi)+\left(1-\xi^{2}\right) \frac{q^{2}}{2 g H} H_{x x}+(\xi-1) \frac{q^{2} Y_{x}}{g H^{2}} H_{x} \\
& +(1-\xi) \frac{q^{2}}{g H} Y_{x x}+\left(\xi^{2}-1\right) \frac{q q_{x x}}{2 g}+\left(1-\xi^{2}\right) \frac{q_{x}^{2}}{2 g}+(1-\xi) \frac{q Y_{x} q_{x}}{g H} .
\end{aligned}
$$

The right-hand side (RHS) of the above equation consists of the hydrostatic and dynamic pressure terms, and the change in pressure as a result of the spatially-varied discharge. The last three terms make the above equation completely different from the pressure equations of $\mathrm{Ze}$ rihun and Fenton (2006) and Fenton and Zerihun (2007). For a gradually-varied flow in a mild-slope channel with a spatially-varied discharge $\left(H_{x x}=Y_{x x}=H_{x} Y_{x} \tilde{=} 0\right)$, Eq. (8) simplifies to a lower-order form of a pressure distribution equation for a non-uniform discharge, i.e.,

$$
\frac{p}{\rho g}=H(1-\xi)+\left(\xi^{2}-1\right) \frac{q q_{x x}}{2 g}+\left(1-\xi^{2}\right) \frac{q_{x}^{2}}{2 g}+(1-\xi) \frac{q Y_{x} q_{x}}{g H} .
$$

In the case of an open-channel flow with a spatially-constant discharge $\left(q_{x}=q_{x x}=0\right)$, Eq. (9) reduces to a well-known hydrostatic pressure equation. Setting $\xi$ equal to zero in Eq. (8) yields the following equation to predict the bed pressure:

$$
\frac{p_{b}}{\rho g}=H+\frac{q^{2}}{g H}\left(\frac{H_{x x}}{2}+Y_{x x}\right)-\frac{q^{2} Y_{x}}{g H^{2}} H_{x}-\frac{q q_{x x}}{2 g}+\frac{q_{x}}{g}\left(\frac{q_{x}}{2}+\frac{q Y_{x}}{H}\right),(10)
$$

where $p_{b}$ is the bed pressure. In the above equations, the subscript $x$ denotes a partial differentiation with respect to the streamwise horizontal axis.

The pressure gradient term in Eq. (1) is evaluated by differentiating Eq. (8) with respect to $x$ and then integrating the result with respect to $y$ over the depth. Substituting the integrated expression into Eq. (1) and re-arranging the terms gives the following equation for a steady, spatially-varied flow in a rectangular channel after some manipulation using the relationships $\partial A / \partial t=B \partial H / \partial t=0$ and $q_{L} / B=q_{x}$ :

$$
\begin{aligned}
& \frac{q^{2}}{3} H_{x x x}+\frac{2 q q_{x}}{3} H_{x x}+\left(g H-\beta \frac{q^{2}}{H^{2}}-\frac{q^{2}}{2 H} Y_{x x}\right) H_{x}+\frac{q^{2}}{2} Y_{x x x} \\
& +\left(\frac{3 q q_{x}}{2}+\frac{q^{2} Y_{x}}{H}\right) Y_{x x}+\left(g H-\frac{q_{x}^{2}}{2}+\frac{q_{x} Y_{x} q}{H}\right) Y_{x}+\left(\frac{q_{x} H}{3}-\frac{q H_{x}}{3}\right) q_{x x} \\
& +\left(2 \beta \frac{q}{H}-\beta_{L} U_{L}\right) q_{x}+\frac{f P_{w} q^{2}\left(1+Y_{x}^{2}\right)}{8 H^{2} B}=0
\end{aligned}
$$


where $B$ is the width of the channel. The eighth term on the left-hand side of this equation, i.e., $\left(2 \beta q / \mathrm{H}-\beta_{L} U_{L}\right)$, depends on the magnitudes of the change in momentum of the main flow and of the momentum of the inflow or outflow. In the case of spatially-varied flow problems, where the lateral inflow enters in the direction normal to the longitudinal axis of the side channel, the contribution of the inflow momentum flux is nil.

Equation (11) is a depth-averaged Boussinesq-type equation for steady, 2D spatially-varied flows with increasing discharges or a lateral outflow due to a side weir. It incorporates spatially-varied discharge terms, which come from a modified vertical velocity profile for fully satisfying the kinematic boundary conditions. Contrary to this, the flow equations of Zerihun and Fenton (2006) and Fenton and Zerihun (2007) do not include these terms and are valid only for flows with spatially-constant discharges. In the case of hydrostatic free-surface flows with spatially-varied discharges, the free-surface streamline and bed curvatures terms vanish. Under this flow condition, Eq. (11) reduces to a shallow-flow equation (Chow, 1959), i.e.,

$$
\begin{aligned}
& \left(g H-\beta \frac{q^{2}}{H^{2}}\right) H_{x}+g H Y_{x}+\left(2 \beta \frac{q}{H}-\beta_{L} U_{L}\right) q_{x} \\
& +\frac{f P_{w} q^{2}}{8 H^{2} B}=0 .
\end{aligned}
$$

Equations (11) and (12) will be used in this study for analyzing spatially-varied open-channel flows with considerable streamline vertical curvatures. As described above, Eq. (11) is capable of analyzing the transition of a flow from a subcritical to a supercritical state, which is caused by streamline vertical curvatures as well as by a lateral inflow or outflow. It is applicable to flow problems with moderately sloped and curved streamlines. For the case of spatially-varied flows in steep-slope channels, flow models that incorporate the effects of a hydraulically steep slope might be extended and used (e.g., Berger, 1992; Steffler and Jin, 1993; Zerihun, 2016; Darvishi et al., 2017). The numerical solutions of the two equations will be compared with the measurements in order to assess quantitatively the contributions of the non-hydrostatic terms of the governing equation of the present study. Eqs. (11) and (12) will be referred to hereafter as the non-hydrostatic flow (NHF) model and the shallow-flow (SF) model, respectively.

\section{NUMERICAL SOLUTION PROCEDURE}

To examine the applicability of the proposed non-hydrostatic depth-averaged model to open-channel flows with a spatially-varied discharge, numerical solutions were obtained for such types of flow problems using an implicit finite-difference scheme; the results were compared with the experimental data. In the numerical method, the spatial derivatives of the bed topography were evaluated analytically from the known bed profile equations. As the flow depth at node $j$ in Eq. (11) was unknown, its spatial derivatives were computed numerically by using the following finite-difference equations (Bickley, 1941):

$$
\begin{gathered}
H_{x, j}=\frac{1}{2 \Delta x}\left(H_{j+1}-H_{j-1}\right), \\
H_{x x, j}=\frac{1}{(\Delta x)^{2}}\left(H_{j-1}-2 H_{j}+H_{j+1}\right), \\
H_{x x x, j}=\frac{1}{(\Delta x)^{3}}\left(-H_{j-1}+3 H_{j}-3 H_{j+1}+H_{j+2}\right),
\end{gathered}
$$

where $H_{x, j}, H_{x x, j}$ and $H_{x x x, j}$ are the first, second and third derivatives, respectively, which were evaluated at node $j$; and $\Delta x$ is the size of the step. In order to minimize numerical errors due to spatial discretization, the size of the step was kept between $0.6 \%$ and $2 \%$ of the horizontal length of the computational domain. For computational nodes near the outflow section, these derivative terms were estimated with three-point backward finite differences. Eqs. (13)-(15) were substituted using Eq. (11) for each computational node, which resulted in a system of non-linear implicit algebraic equations. This system of equations, together with the specified boundary conditions, was simultaneously solved using an iterative method, which proceeded from an assumed initial free-surface position. The non-linear algebraic equations were linearized by using the Newton-Raphson method with a numerical Jacobian matrix. The convergence of the numerical solutions was assessed on the basis of the relative change in the solution criterion with a convergence tolerance of $10^{-6}$.

\section{NUMERICAL SIMULATIONS AND DISCUSSION}

A discussion of the simulation results for free-surface flow problems with spatially increasing and decreasing discharges is presented in this section. In both cases, the mean flow characteristics of the spatially-varied flows in the receiving or main channels were analyzed using the proposed model. In this model, the flow resistance was estimated by using the Darcy-Weisbach equation with an explicit form of the Colebrook-White formula (Zigrang and Sylvester, 1982) for the friction factor. Because its value depends solely on the Reynolds number, the computed friction factor varied along the computational nodes. The procedure applied has basically been developed for a free-surface flow with a spatially-constant discharge and is assumed to provide a reasonable approximation for the friction factor of a flow with a spatially-varied discharge. In addition, because of its complexity in terms of a general specification, the value of the momentum correction coefficient was taken as a unity.

In the present study, the elevations of the initial free-surface profile were estimated by linearly interpolating the boundary values at the inflow and outflow sections. Also, all the computational results were presented graphically and expressed in non-dimensional forms such as $x / L, \eta / L$ and $Q / Q_{1}$, where $L$ is the length of the lateral inflow or outflow zone; and $Q_{1}$ is the discharge in the main channel just upstream of the side weir. It is worth noting that the selected experimental data for model validations is free of surface tension and viscosity effects.

\subsection{Side-channel flows with a spatially-increasing discharge}

\subsubsection{Transcritical spatially-varied flows}

The preceding numerical model was applied to analyze a spatially-varied flow in a lateral-spillway channel. The dynamic features of the free-surface flow in such a channel are governed not only by the conditions of the lateral inflow from the spillway but also by the characteristics of the axial channel flow. As described by Hager (2010), spiral currents of one or two vortex cells may be superposed on the main forward flows depending on the level of the tailwater and the lateral-inflow conditions. In the modeling of this type of open-channel flow problem, the usual computational approach is to solve the problem as a $2 \mathrm{D}$ flow problem on a vertical plane by considering the mean flow patterns. Accordingly, the free-surface profile simulation can be performed by computing the cross-sectional average depths along the centerline of the side channel.

The experimental data of Sassoli (1959) for a side-channel flow was invoked to verify the computational results of the NHF model. He conducted a series of experiments in the rectangular, prismatic 
side channels of a considerable bed slope. The crest length of the side-channel spillway was $2.50 \mathrm{~m}$, and the bed slope of the receiving channel varied from $5 \%$ to $15 \%$. No flow was entered at the upstream end, and the inflow to the channel was introduced vertically at a uniform rate. A nearly horizontal free-surface profile was maintained in the transverse direction by a grid structure inserted in the test channels. Under the conditions of the experiment, air entrainment in the side-channel flow was absent.

For spatially-varied flows with a uniform lateral-inflow rate, the discharge increases linearly as $Q(x)=q_{L} x$, in which $q_{L}$ is the lateral inflow per unit length, with $x=0$ at the upstream end of the lateral-inflow zone (see Fig. 2). Since the lateral inflow entered the receiving channel perpendicular to its longitudinal axis, the streamwise momentum flux due to the lateral inflow was zero. For a no-flow boundary condition at the upstream end, Eq. (11) resulted in a horizontal free surface $\left(H_{x, 0}=-Y_{x, 0}\right)$. Additionally, an experimentally determined flow depth at this extreme end and a normal depth at the downstream end, which was computed using the uniform-flow equation, were specified as the boundary conditions. Fig. 3 shows the simulation results for a transcritical flow problem with a spatially-varied discharge. As shown in this figure, the NHF model results correlated well with the experimental data of Sassoli, with a mean relative error of less than $3 \%$. As expected, the effect of channel bed slope is insignificant even for a bed slope of $15 \%$ or $8.5^{\circ}\left(\cos ^{2} 8.5^{\circ}=0.98 \cong 1.0\right)$. Similar investigation results were obtained for curvilinear flows with spatially-constant discharges in moderately sloping channels (see, e.g., Zerihun, 2004). For this spatially-varied flow problem with a non-hydrostatic pressure distribution, the flow transition from a subcritical to a supercritical state was accurately simulated by the NHF model.
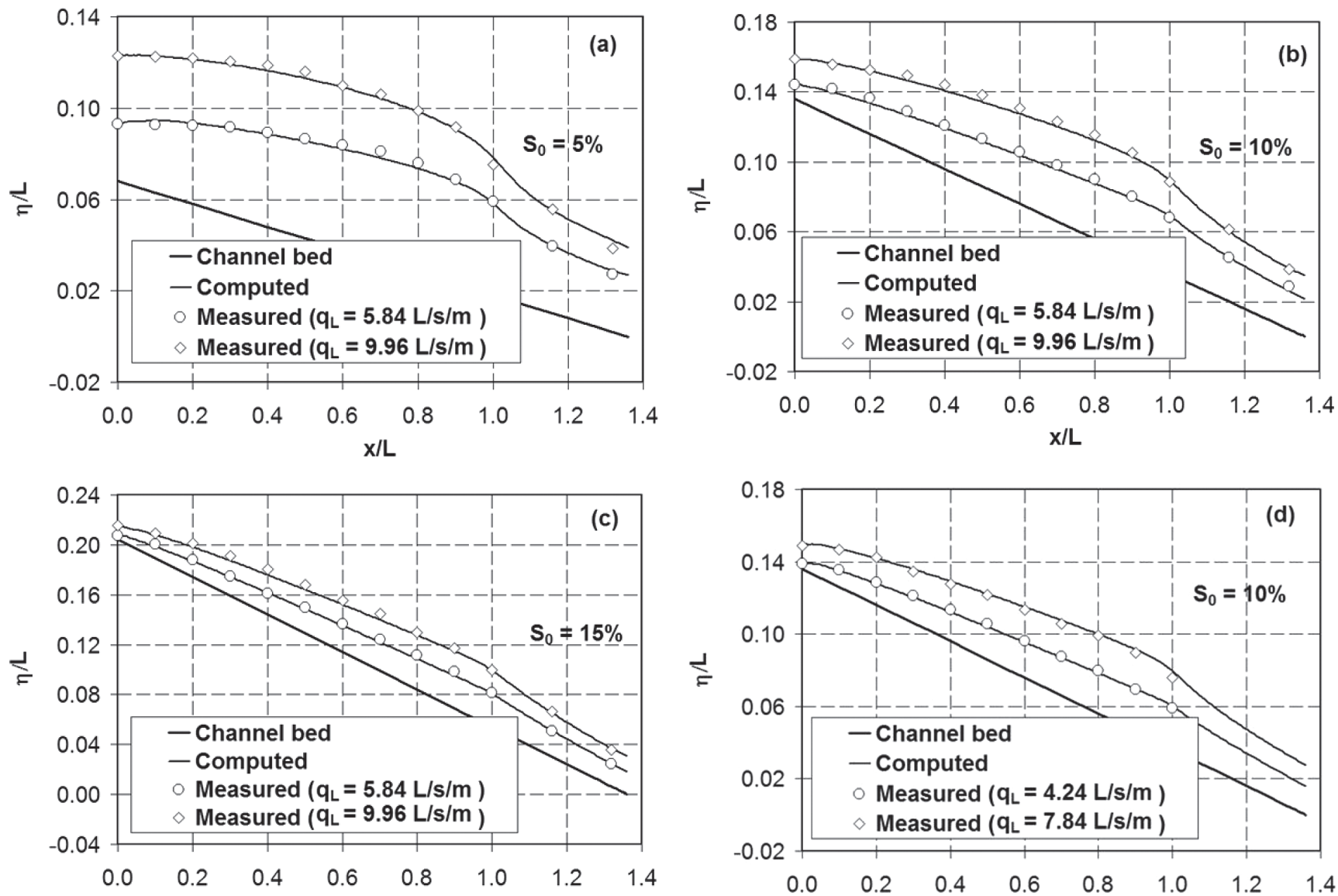

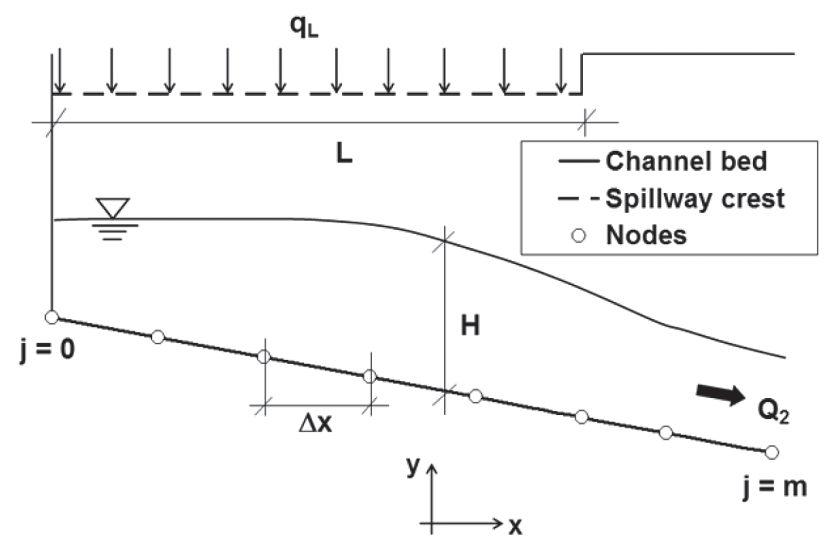

Fig. 2 Definition sketch for a spatially-varied flow in a lateralspillway channel with an upstream dead-end. The discharge in the side channel just downstream of the spillway is indicated by $Q_{2}$

\subsubsection{Subcritical spatially-varied flows}

The proposed NHF model was further investigated by simulating subcritical spatially-varied flows, and the numerical results were compared with the computational results of the SF model and experimental data of Gill (1977). The Gill (1977) experiments were performed in a rectangular-shaped tilting flume $76.2 \mathrm{~mm}$ wide, $250 \mathrm{~mm}$ deep, and $5.0 \mathrm{~m}$ long. He used a perforated Perspex pipe to discharge

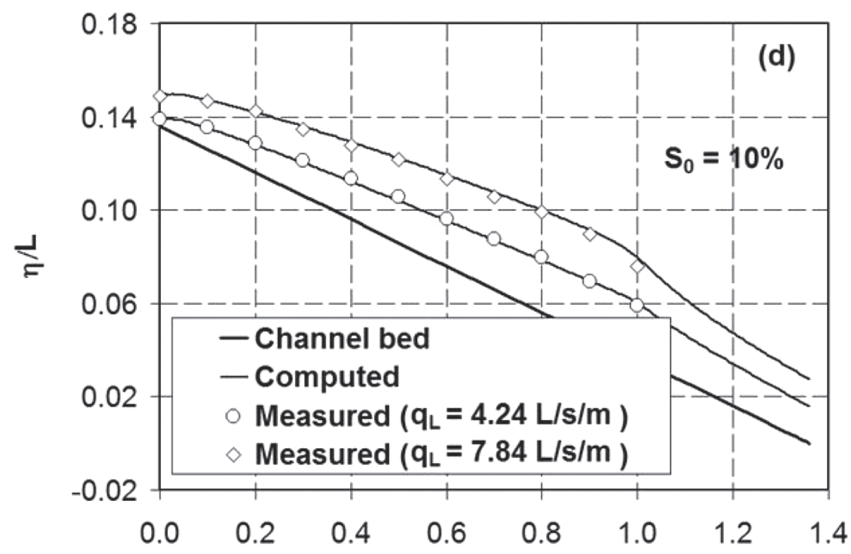

Fig. 3 Free-surface profiles for transcritical flows in side channels with spatially-increasing discharges. The free-surface elevations are computed for a lateral-inflow rate ranging from 4.24 to $9.96 \mathrm{~L} / \mathrm{s} / \mathrm{m}$ and for the cases of mild and moderate channel bed slopes 

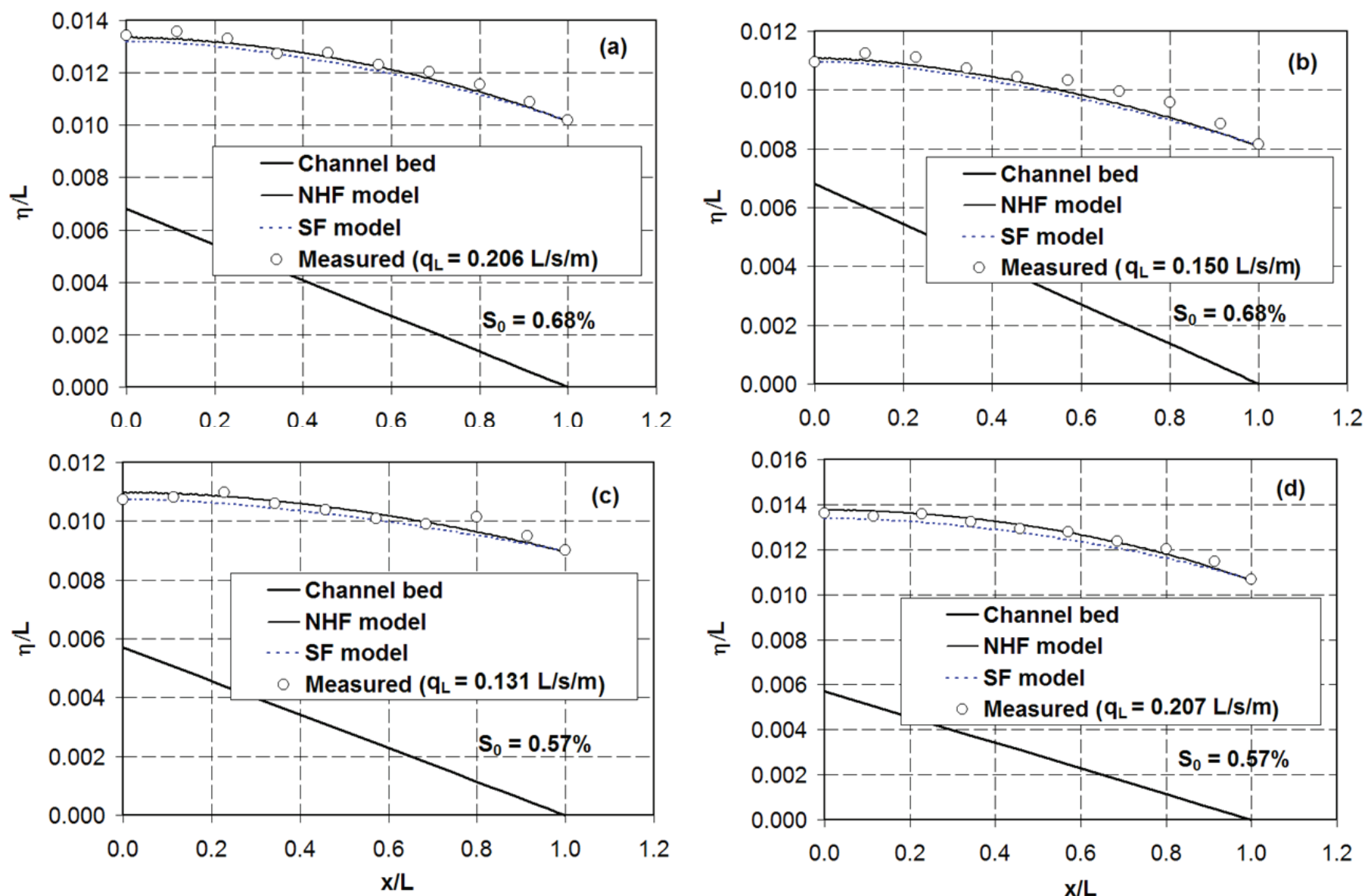

Fig. 4 Free-surface profiles for subcritical spatially-varied flows in side channels with various outflow discharges: (a) $Q_{2}=0.721 \mathrm{~L} / \mathrm{s}$; (b) $Q_{2}=0.525 \mathrm{~L} / \mathrm{s}$; (c) $Q_{2}=0.459 \mathrm{~L} / \mathrm{s}$; and (d) $Q_{2}=0.725 \mathrm{~L} / \mathrm{s}$

the water uniformly along its length into the receiving channel with an upstream dead-end. A tailgate at the downstream end of the channel was used to regulate the depth of the flow within the channel. Similar boundary conditions as in the previous test case were imposed for the solutions of the NHF model, whereas the SF model was solved subject only to a downstream boundary condition. As shown in Fig. 4, the computed free-surface profiles agreed reasonably well with the experimental data. Some discrepancies between the results of the two models and the experimental data can be seen in Fig. 4b. The models underestimated the elevations of the free-surface profile, especially near the outflow section $(0.5<x / \mathrm{L}<0.95)$. For this weakly non-hydrostatic flow problem, the overall quality of the results of the NHF model was marginally better than the results of the SF model The maximum mean relative errors for the NHF and SF models were only $2.6 \%$ and $3.5 \%$, respectively.

\subsection{Free-surface flows with a spatially-decreasing discharge}

As described before, the flow in a channel with the withdrawal of the flow through a side weir is a typical case of a spatially-varied flow with a decreasing discharge. In the vicinity of the side weir, the flow patterns in the main channel tend to be complex and three-dimensional (3D). Depending on the magnitude of the approach flow Froude number, separation of the flow may occur along the opposite side of the channel near the downstream end of the weir. Such a chal- lenging flow phenomenon is treated herein by assuming that the flow conditions are gradually varied in the streamwise direction. Hence, the spatially-varied flow problem can be approximately solved by a non-hydrostatic approach in which the transverse variations of the free-surface profile and velocity distribution are ignored.

\subsubsection{Discharge coefficients}

The computation of a discharge over a side weir becomes more complicated as the flow velocity through the side weir is at an oblique angle rather than at right angles to the crest axis, as in the case of a transverse weir flow. By assuming a constant specific energy across a weir, however, De Marchi (1934) proposed the following discharge equation for a rectangular side weir:

$$
Q_{x}=-\frac{2}{3} C_{D} \sqrt{2 g\left(H-H_{w}\right)^{3}},
$$

where $C_{D}$ is the discharge coefficient; $H_{w}$ is the height of the weir; and $Q_{x}$ is the discharge per unit length along the side weir. Fig. 5 illustrates a schematic diagram for side-weir flow problems with subcritical flow conditions. It has been shown by several studies (Ranga Raju et al., 1979; Borghei et al., 1999; Paris et al., 2012) that the assumption of a constant specific energy is acceptable for subcritical flow conditions. A dimensional analysis demonstrates that $C_{D}$ is a function of the following parameters provided that the scale effects are excluded: 


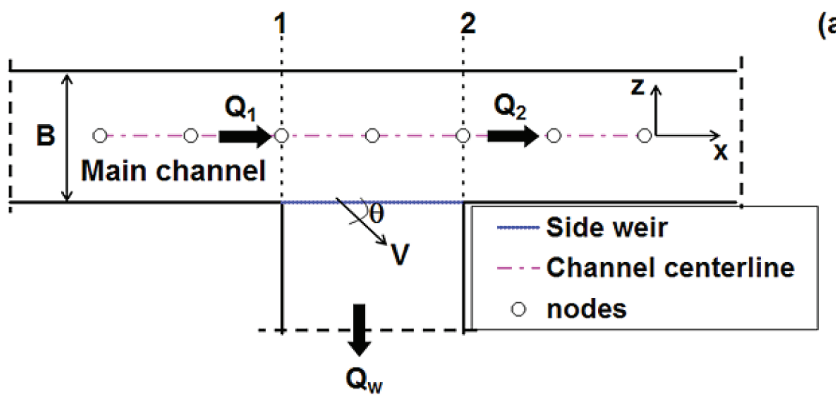

(a)

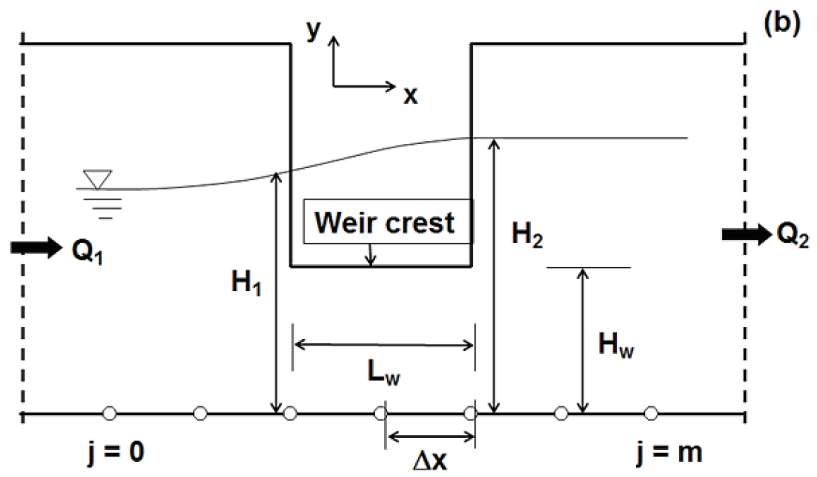

Fig. 5 Definition sketch of a free flow over a rectangular side weir: (a) plan; and (b) longitudinal section. The lateral-outflow discharge $\left(Q_{w}\right)$ is indicated along with the flow depth at the downstream end of the weir $\left(\mathrm{H}_{2}\right)$

$$
C_{D}=f_{n}\left(F_{1}, \frac{L_{w}}{B}, \frac{H_{w}}{H_{1}}\right)
$$

where $L_{w}$ is the length of the side weir; $H_{l}$ is the depth of the flow at the upstream end of the side weir; and $F_{l}$ is the Froude number of the approaching flow. It was reported by Borghei et al. (1999) that the effect of a channel bed slope on $C_{D}$ is negligible for subcritical flow conditions. For modeling the mean flow characteristics of a spatially-varied flow in a main channel, an appropriate expression for the side-weir discharge coefficient is developed here.

To include the effects of all the parameters given by Eq. (17), the following discharge coefficient equation is proposed:

$$
C_{D}=k_{1}+k_{2} F_{1}^{2}+k_{3}\left(\frac{L_{w}}{B}\right)^{k_{4}}+k_{5}\left(1-\frac{H_{w}}{H_{1}}\right)^{k_{6}},
$$

where $k_{1}-k_{6}$ are unknown constants. These constants are determined from the experimental data using a data modeling technique. Differentiating Eq. (16) with respect to $x$ and ignoring the contribution of the $d C_{D} / d x$ term, which is very small compared to the other terms, gives

$$
Q_{x x}=-C_{D} \sqrt{2 g\left(H-H_{w}\right)}\left(H_{x}-H_{w, x}\right)
$$

where $H_{w, x}$ is the slope of the crest of the weir.

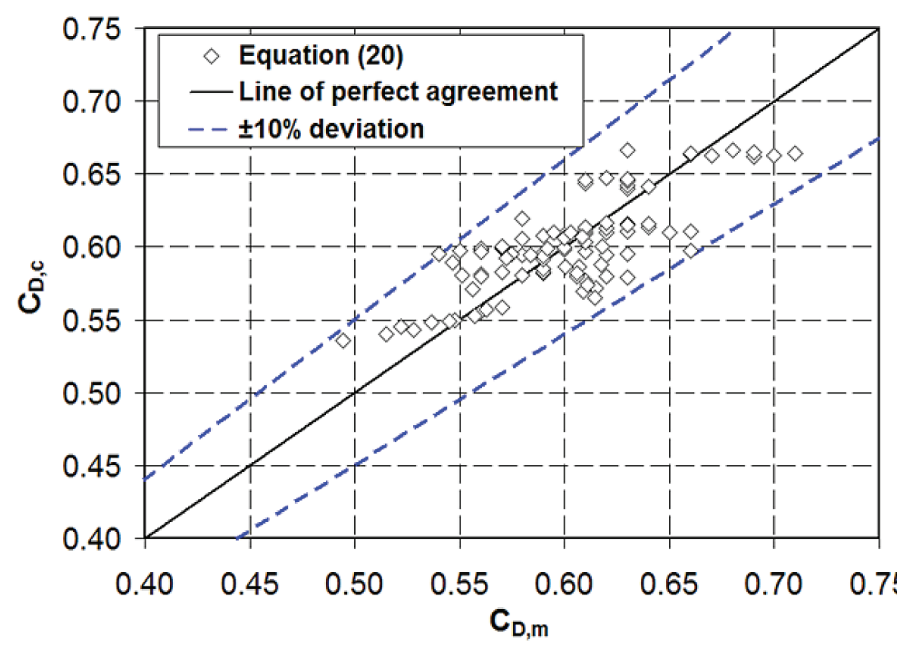

Fig. 6 Comparison of the predicted and measured discharge coefficients for flows over rectangular side weirs under free-flow conditions
In the present study, a simple optimization method was employed to determine the above unknown constants with the experimental data of Emiroglu et al. (2011) and Novak et al. (2013). Both experiments were conducted in rectangular cross-section channels to explore the discharge characteristics of rectangular side weirs under free-flow conditions. For the selected experimental data, values of the various parameters range in such a way that: (i) $0.2 \leq F_{1} \leq 0.80$; (ii) $0.22 \leq L_{w} / B \leq 3.33$; and (iii) $0.35 \leq H_{w} / H_{1} \leq 0.89$. The discharge coefficients for all the runs were computed using Eq. (16) with the measured values of the weir discharge and the corresponding flow depths at a mid-point location of the side weir. These values were used to estimate the errors corresponding to the assigned trial values of the constants. Using the Solver module in the Excel spreadsheet package, the sum of the square of the errors was minimized to yield the following best-fit results of Eq. (18):

$$
C_{D}=0.61-0.033 F_{1}^{2}+0.146\left(\frac{L_{w}}{B}\right)^{0.401}-0.151\left(1-\frac{H_{w}}{H_{1}}\right)^{-0.10} .
$$

The correlation coefficient and the root mean square error leading to Eq. (20) are 0.80 and $2.54 \%$, respectively. If the contribution of the last two terms on the RHS of the equation is ignored, then a theoretical value of $C_{D} \cong 0.61$ can be extrapolated from this equation for $F_{1} \rightarrow 0$. The calibration results, which compare the computed discharge coefficient, $C_{D, c}$, with the experimental value, $C_{D, m}$, is shown in Fig. 6.

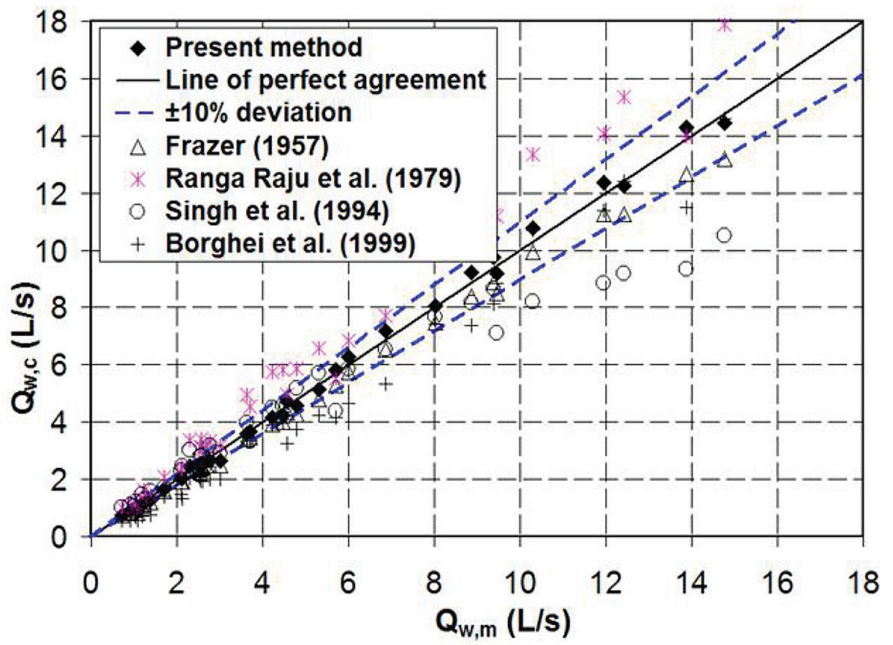

Fig. 7 Measured discharges $\left(Q_{w, m}\right)$ compared with simulated discharges $\left(Q_{w, c}\right)$ computed by various methods for lateral outflows over side weirs under free-flow conditions 

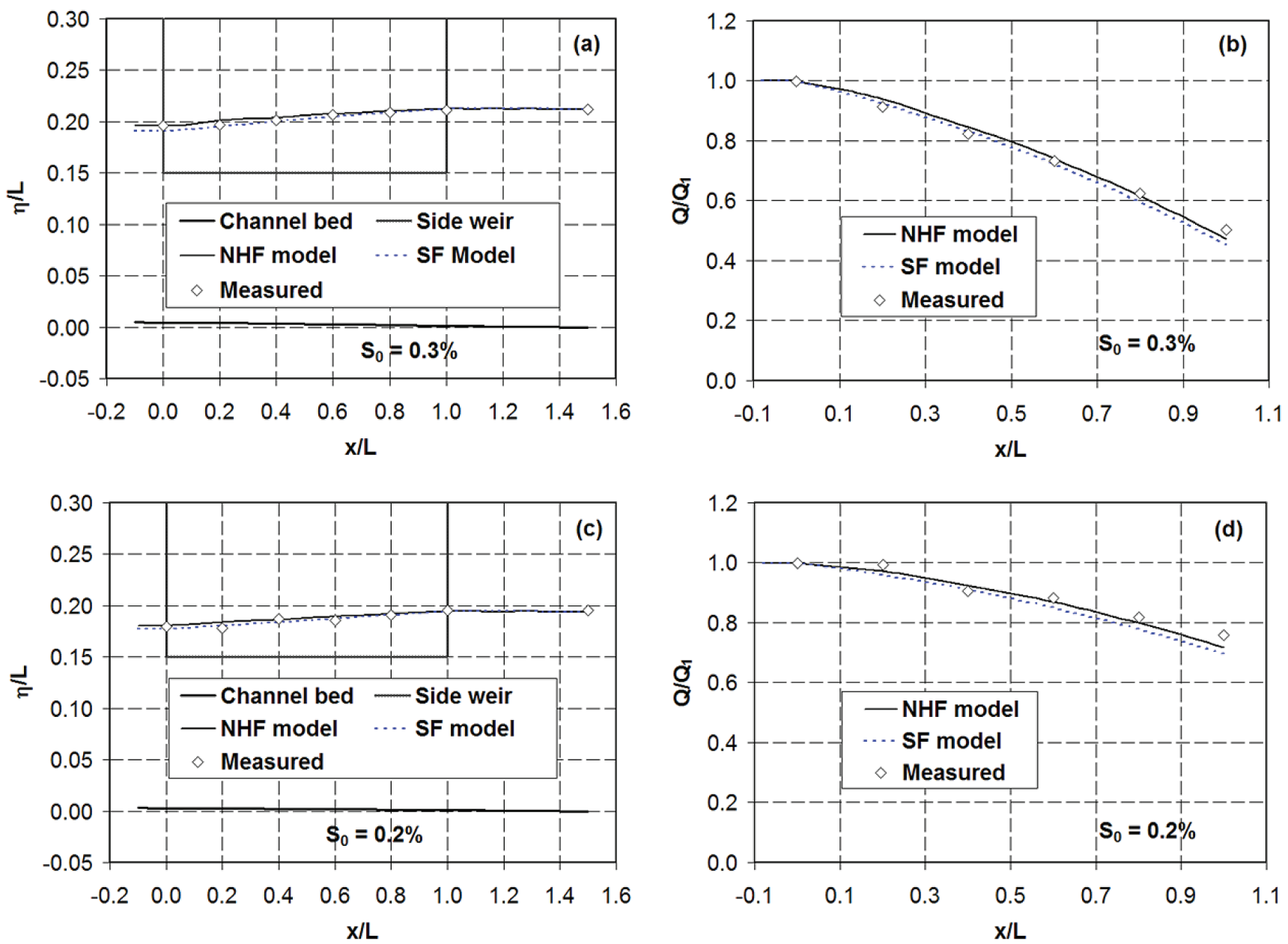

Fig. 8 Axial free-surface profiles and variation of the main-channel discharge for a quasi-hydrostatic flow with a spatially-decreasing discharge. The Froude numbers of the incoming flow for (a) and (b) and for (c) and (d) are 0.496 and 0.562, respectively

The computed discharge based on Eq. (20) was also compared with the experimental data of Carballada (1979) and the results of the previous empirical equations proposed by Frazer (1957), Ranga Raju et al. (1979), Singh et al. (1994), and Borghei et al. (1999). Carballada (1979) conducted a series of experiments for the flows over side weirs using a rectangular test flume. The flume was $254 \mathrm{~mm}$ wide and $432 \mathrm{~mm}$ deep. Rectangular side weirs of different heights and lengths were tested for the various inflow discharges. As shown in Fig. 7, the agreement between the results of the present method and the experimental data was relatively close, with a mean relative error of $4 \%$. For the higher discharges, the results from the equations of Ranga Raju et al. (1979) and Singh et al. (1994) fell out of the region of $\pm 10 \%$.

\subsubsection{Results of the numerical model}

The results of the NHF and SF models were compared with the experimental data of Hager (1982) for subcritical side-weir flows. The experiments were conducted in a rectangular cross-section channel with a width of $30 \mathrm{~cm}$ and a length of $570 \mathrm{~cm}$. The bed slope of the main channel varied between $-0.5 \%$ and $2 \%$. Rectangular side weirs having a lateral opening length of $100 \mathrm{~cm}$ and different heights were tested for various inflow discharges. The free-surface profile and main-channel discharge measurements of the selected runs were used to validate the numerical models.

For the solutions of the NHF model, the depths of the flow at the two extreme ends of the computational domain were specified as the boundary conditions. At the upstream end, the free-surface slope was computed from the specified flow depth using the gradually-varied flow equation. In addition, the lateral-outflow angle for the side-weir flow was estimated with the equation proposed by Hager (1987). Identical boundary conditions as in the previous test case were used for the SF model. Fig. 8 compares the computed free-surface and main-channel discharge profiles with the experimental data. The validation results showed a good correlation between the results of the NHF model and the experimental data throughout the computational domain for the centerline free-surface profile along the main channel. The results of the SF model near the upstream edge of the side weir slightly departed from the measurements due to the curvature of the local streamline. A minor discrepancy between the predicted and measured values of the main-channel discharge was observed in the flow region near the downstream end of the side weir $(x / L>0.7)$. This discrepancy is attributed to the 3D characteristics of the flow in this region, which cannot be accurately portrayed by these models. In general, the overall quality of the numerical solutions for the main-channel discharge was satisfactory, with mean relative errors of less than $4 \%$ for both models.

The performance of the two models was further examined by modeling a quasi-hydrostatic flow problem, and the results were validated using the experimental data of Emiroglu et al. (2011). As can be seen from Fig. 9, the results of both models for the free-surface profile showed good agreement with the experimental data, and no substantial differences can be seen between the results of the two models for this nearly hydrostatic flow situation. At the upstream and 

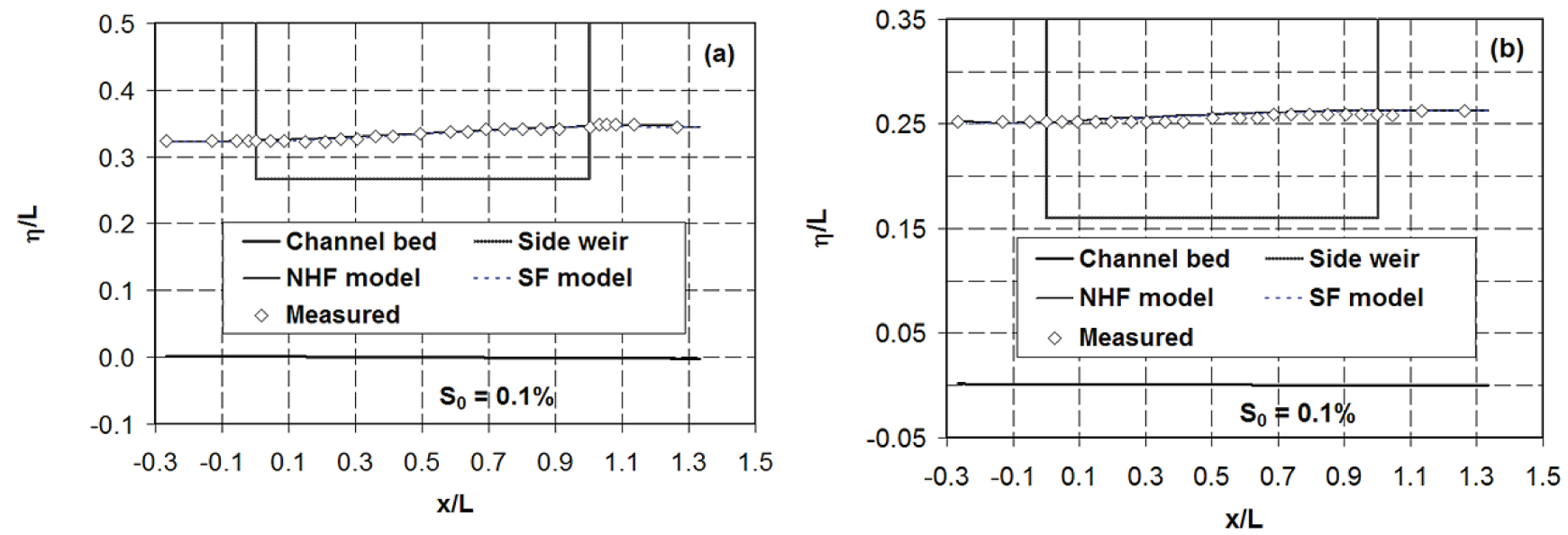

Fig. 9 Axial free-surface profiles for a spatially-varied open-channel flow with a decreasing discharge: (a) $F_{1}=0.63 ;$ and (b) $F_{1}=0.28$

downstream ends of the lateral-outflow zone, the SF model gave results with a continuous free-surface slope. As expected, the effect of a non-hydrostatic pressure distribution is not quite so significant for these flow problems with subcritical flow conditions in the side-weir region.

The free-surface and main-channel discharge profiles of the preceding spatially-varied flow problems were predicted reasonably well by the proposed non-hydrostatic depth-averaged model. For these problems, the curvilinearity of the streamlines is not so pronounced compared to the 2D structure of the flow over bottom intake racks (see, e.g., Mostkow, 1957; Nakagawa, 1969). The results of the analysis revealed that this type of model is suitable for analyzing curvilinear flow problems with a lateral flow into or leaving (due to a side weir) a moderately sloping channel. It is also apparent that the flow depth at the upstream end of the weir is an important parameter for a side-weir flow study related to discharge characteristics. The computational results of the free-surface profile (see Figs. 8 and 9) confirmed that this parameter may be predicted with a reasonable degree of accuracy by excluding the non-hydrostatic terms from the NHF model. In other words, it is sufficient to apply the SF model for analyzing the problem of a side-weir flow with subcritical flow conditions.

\section{SUMMARY AND CONCLUSIONS}

Herein, a simple method, which utilizes the Boussinesq theory, was applied to develop a depth-averaged spatially-varied flow model for analyzing the mean flow characteristics of a free-surface flow with a spatially increasing or decreasing discharge. The model takes into account the effects of a spatially-varied discharge and a non-hydrostatic pressure distribution and overcomes the accuracy problem of the shallow-flow model when simulating a spatially-varied flow with strong curvilinear effects. The proposed model was solved using an implicit finite-difference scheme and then used to simulate the transcritical flow in a lateral-spillway channel and the subcritical flow in a main channel fitted with side weirs. The computational results for the steady-state flows were compared with the results of the shallow-flow model and the experimental data from the literature.

For transcritical spatially-varied flow problems, the proposed model accurately simulated the flow transition from a subcritical to a supercritical state. Additionally, it correctly emulated the free-surface profiles of a subcritical flow problem with a spatially-increasing discharge. For this weakly-curved flow problem, the overall quality of the results of the non-hydrostatic model was marginally better than the results of the shallow-flow model.
As a part of the investigation, an empirical discharge coefficient equation for a subcritical side-weir flow was proposed by analyzing the experimental data using an optimization method. The analysis results attested that the empirical equation was capable of predicting the side-weir discharge under free-flow conditions accurately, with a mean relative error of $4 \%$. In the case of a spatially-varied flow with a decreasing discharge, the numerical results of both models for the free-surface profile and main-channel discharge distribution were favorable compared with the experimental data. A minor discrepancy between the predicted and measured values of the main-channel discharge was observed in the flow region near the downstream end of the side weir $(x / L>0.7)$. This discrepancy is attributed to the $3 \mathrm{D}$ characteristics of the flow in this region, which cannot be portrayed accurately by these models. Furthermore, the results of the investigation confirmed that it was adequate to apply the shallow-flow model for analyzing the problem of a subcritical side-weir flow with a nearly hydrostatic flow situation. The numerical model described in the present study is a promising tool for solving real-life problems related to the design of a main or receiving channel and the analysis of spatially-varied flow processes in a prismatic open channel that conveys surface runoff. 


\section{REFERENCES}

Balmforth, D. J. - Sarginson, E. J. (1983) The effects of curvature in supercritical side-weir flow. J. Hydr. Res., 21(5), 333-343.

Berger, R. C. (1992) Free-Surface Flow over Curved Surfaces. Ph.D. Thesis, University of Texas, Austin, TX, USA.

Bickley, W. G. (1941) Formulae for numerical differentiation. Math. Gaz., 25(263), 19-27. https://doi.org/10.2307/3606475

Borghei, S. M. - Jalili, M. R. - Ghodsian, M. (1999) Discharge coefficient for sharp-crested side weir in subcritical flow. J. Hydr. Eng., 125(10), 1051-1056.

Bremen, R. - Hager, W. H. (1989) Experiments in side-channel spillways. J. Hydr. Eng., 115(5), 617-635.

Camp, T. R. (1940) Lateral spillway channels. Trans. ASCE, 195(1), 606-617.

Carballada, L. B. (1979) Some Characteristics of Lateral Flow. Ph.D. Thesis, Concordia University, Montreal, QC, Canada.

Castro-Orgaz, O. - Hager, W. H. (2011) Spatially-varied open-channel flow equations with vertical inertia. J. Hydr. Res., 49(5), 667-675.

Chow, V. T. (1959) Open-Channel Hydraulics. McGraw-Hill, New York, NY, USA, 331.

Darvishi, E. - Fenton, J. D. - Kouchakzadeh, S. (2017) Boussinesq equations for flows over steep slopes and structures. J. Hydr. Res., 55(3), 324-337.

De Marchi, G. (1934) Saggio di teoria del funzionamento degli stramazzi laterali (Essay of the performance of lateral weirs). L'Energia Elettrica, 11(11), 849-860 [in Italian].

Emiroglu, M. E. - Agaccioglu, H. - Kaya, N. (2011) Discharging capacity of rectangular side weirs in straight open channels. Flow Meas. Instrum., 22, 319-330.

Fareny, H. S. - Markus, A. (1962) Side-channel spillway design. J. Hydr. Div., 88(HY3), 131-154.

Favre, H. (1933) Contribution à l'étude des courants liquides (Contribution to the study of liquid flows). Rascher et Cie, Zürich [in French].

Fenton, J. D. - Zerihun, Y. T. (2007) A Boussinesq approximation for open-channel flow. In: Proceedings of the 32nd Congress, IAHR, Venice, Italy, 2-6 July.

Frazer, W. (1957) The behavior of side weirs in prismatic rectangular channels. Proc. Instn. Civ. Eng. London, England, 6(2), 305-328.

Gill, M. A. (1977) Perturbation solution of spatially-varied flow in open channels. J. Hydr. Res., 15(4), 337-350.

Hager, W. H. (1982) Die Hydraulik von Verteilkanälen 1: Theorie der Verteilkanäle (Hydraulics of Distribution Channels 1: Theo$r y)$. DScTech Thesis, Mitteilungen derVersuchsanstalt für Wasserbau, Hydrologie und Glaziologie, ETH-Zürich, D. Vischer, Ed., Nr. 55 , Zürich, Switzerland [in German].

Hager, W. H. (1983) Open-channel hydraulics of flows with increasing discharge. J. Hydr. Res., 21(3), 177-193.
Hager, W. H. - Volkart, P. U. (1986) Distribution channels. J. Hydr. Eng., 112(10), 935-952.

Hager, W. H. (1987) Lateral outflow over side weirs. J. Hydr. Eng., 113(4), 491-504.

Hager, W. H. - Edder, O. - Rappaz, J. (1988) Streamline curvature effects in side-channel spillway flow. Acta Mech., 72(1-2), 95-110.

Hager, W. H. (2010) Wastewater Hydraulics-Theory and Practice. 2nd ed.; Springer-Verlag: Berlin Heidelberg, Germany, 601.

Hinds, J. (1926) Side-channel spillway: Hydraulic theory, economic factors, and experimental determination of losses. Trans. ASCE, 89, 881-927.

Kouchakzadeh, S. - Vatankhah, A. R. - Townsend, R. D. (2001) A modified perturbation solution procedure for spatially-varied flows. Can. Water Resour. J., 26(3), 399-416.

Li, W. H. (1955) Open channels with non-uniform discharge. Trans. ASCE, 120(1), 255-274.

Liggett, J. A. (1959) Unsteady Open-Channel Flow with Lateral Inflow. Technical Report NO. 2, Department of Civil Engineering, Stanford University, Stanford, CA, USA.

Maranzoni, A. - Pilotti, M. - Tomirotti, M. (2017) Experimental and numerical analysis of side-weir flows in a converging channel. J. Hydr. Eng., 143(7), DOI: 10.1061/(ASCE)HY.19437900.0001296.

Montes, J. S. (1998) Hydraulics of Open-Channel Flow. ASCE Press, Reston, VA, USA, 300-301.

Mostkow, M. A. (1957) Sur le calcul des grilles de prise d'eau (Theoretical study of a bottom type water intake). La Houille Blanche, 12(4), 570-580 [in French].

Nakagawa, H. (1969) On hydraulic performance of bottom diversion works. Bulletin of the Disaster Prevention Research Institute, Kyoto University, 18(3), 29-48. Retrieved from http://hdl.handle. net/2433/124757.

Novak, G. - Kozelj, D. - Steinman, F. - Bajcar, T. (2013) Study of flow at side weir in narrow flume using visualization techniques. Flow Meas. Instrum., 29, 45-51.

Paris, E. - Solari, L. - Bechi, G. (2012) Applicability of the De Marchi hypothesis for side-weir flow in the case of movable beds. J. Hydr. Eng., 138(7), 653-656.

Ranga Raju, K. G. - Prasad, B. - Gupta, S. K. (1979) Side weir in rectangular channel. J. Hydr. Div., 105 (HY5), 547-554.

Sassoli, F. (1959) Canali collettori laterali a forte pendenza (Side channels of a large bottom slope). L'Energia Elettrica, 36(1), 26-39 [in Italian].

Singh, R. - Manivannan, D. - Satyanarayana, T. (1994) Discharge coefficient of rectangular side weirs. J. Irrig. Drain. Eng., 120(4), 814-819.

Steffler, P. M. - Jin, Y. (1993) Depth averaged and moment equations for moderately shallow free-surface flow. J. Hydr. Res., $31(1), 5-17$. 
White, F. M. (2011) Fluid Mechanics. 7th ed., McGraw-Hill, New York, NY, USA, 242.

Yen, B. C. - Wenzel, H. G. (1970) Dynamic equation for steady spatially-varied flow. J. Hydr. Div., 96(HY3), 801-814.

Yen, B. C. (1971) Spatially-varied open-channel flow equations. Water Resources Center, Research Report No. 51, University of Illinois, Urbana, IL, USA.

Yen, B. C. (1973) Open-channel flow equations revisited. J. Eng. Mech. Div., 99(EM5), 979-1009.

Zerihun, Y. T. (2004) A One-Dimensional Boussinesq-Type Momentum Model for Steady Rapidly Varied Open-Channel Flows. $\mathrm{Ph} . \mathrm{D}$. Thesis, Department of Civil and Environmental Engineering, The University of Melbourne, Australia.
Zerihun, Y. T. - Fenton, J. D. (2006) One-dimensional simulation model for steady transcritical free-surface flows at short length transitions. Adv. Water Resour., 29(11), 1598-1607.

Zerihun, Y. T. (2016) Modeling free-surface flow with curvilinear streamlines by a non-hydrostatic model. J. Hydrol. Hydromech., 64(3), 281-288.

Zigrang, D. J. - Sylvester, N. D. (1982) Explicit approximations to the solution of Colebrook's friction factor equation. AIChE J., 28(3), 514-515. 